\title{
Fixed points and quadratic equations connected with homomorphisms and derivations on non-Archimedean algebras
}

\author{
Madjid Eshaghi Gordji ${ }^{1,2^{*}}$, Hamid Khodaei ${ }^{1,2}$, Razieh Khodabakhsh ${ }^{2}$ and Choonkil Park ${ }^{3^{*}}$
}

\begin{tabular}{l}
\hline "Correspondence: \\
meshaghi@semnan.ac.ir; \\
baak@hanyang.ac.kr \\
'Department of Mathematics, \\
Semnan University, P.O. Box \\
35195-363, Semnan, Iran \\
3Department of Mathematics, \\
Research Institute for Natural \\
Sciences, Hanyang University, Seoul, \\
133-791, Republic of Korea \\
Full list of author information is \\
available at the end of the article
\end{tabular}

available at the end of the article

\begin{abstract}
We apply the fixed point method to prove the stability of the systems of functional equations

$$
\begin{aligned}
& \left\{\begin{array}{l}
f(x y)=f(x) f(y) ; \\
f(a x+b y)+f(a x-b y)=2 a^{2} f(x)+2 b^{2} f(y),
\end{array}\right. \\
& \left\{\begin{array}{l}
f(x y)=x^{2} f(y)+f(x) y^{2} ; \\
f(a x+b y)+f(a x-b y)=2 a^{2} f(x)+2 b^{2} f(y)
\end{array}\right.
\end{aligned}
$$

on non-Archimedean Banach algebras. Moreover, we give some applications of our results in non-Archimedean Banach algebras over $p$-adic numbers.
\end{abstract}

MSC: 34K36; 46S40; 47S40; 39B82; 39B52; 26E50

Keywords: fixed point approach; non-Archimedean algebra; quadratic homomorphism; quadratic derivation; stability

\section{Introduction and preliminaries}

The story of the stability of functional equations dates back to 1925 when a stability result appeared in the celebrated book by Gy. Pólya and G. Szegö [1]. In 1940, Ulam [2] posed the famous Ulam stability problem which was partially solved by Hyers [3] in the framework of Banach spaces. Later, Aoki [4] considered the stability problem with unbounded Cauchy differences. In 1978, Th. M. Rassias [5] provided a generalization of the Hyers' theorem by proving the existence of unique linear mappings near approximate additive mappings. Găvruta [6] obtained a generalized result of Th. M. Rassias' theorem which allows the Cauchy difference to be controlled by a general unbounded function. On the other hand, J. M. Rassias [7-10] considered the Cauchy difference controlled by a product of different powers of norm. However, there was a singular case; for this singularity, a counterexample was given by Gǎvruta [11].

Bourgin [12] proved the stability of ring homomorphisms in two unital Banach algebras. Badora [13] gave a generalization of the Bourgin's result. The stability result concerning derivations on operator algebras was first obtained by Šemrl [14]. In [15], Badora proved the stability of functional equation $f(x y)=x f(y)+f(x) y$, where $f$ is a mapping on normed algebra $A$ with unit.

(0) 2012 Eshaghi Gordji et al.: licensee Springer. This is an Open Access article distributed under the terms of the Creative Commons Attribution License (http://creativecommons.org/licenses/by/2.0), which permits unrestricted use, distribution, and reproduction in any medium, provided the original work is properly cited. 
Let $\mathcal{A}, \mathcal{B}$ be two algebras. A mapping $f: \mathcal{A} \rightarrow \mathcal{B}$ is called a quadratic homomorphism if $f$ is a quadratic mapping satisfying $f(x y)=f(x) f(y)$ for all $x, y \in \mathcal{A}$. For instance, let $\mathcal{A}$ be commutative. Then the mapping $f: \mathcal{A} \rightarrow \mathcal{A}$, defined by $f(x)=x^{2}(x \in \mathcal{A})$, is a quadratic homomorphism.

A mapping $f: \mathcal{A} \rightarrow \mathcal{A}$ is called a quadratic derivation if $f$ is a quadratic mapping satisfying $f(x y)=x^{2} f(y)+f(x) y^{2}$ for all $x, y \in \mathcal{A}$. For instance, consider the algebra of $2 \times 2$ matrices

$$
\mathcal{A}=\left\{\left[\begin{array}{cc}
c_{1} & c_{2} \\
0 & 0
\end{array}\right]: c_{1}, c_{2} \in \mathbb{C}(\text { complex field })\right\} .
$$

Then it is easy to see that the mapping $f: \mathcal{A} \rightarrow \mathcal{A}$, defined by $f\left(\left[\begin{array}{cc}c_{1} & c_{2} \\ 0 & 0\end{array}\right]\right)=\left[\begin{array}{cc}0 & c_{2}^{2} \\ 0 & 0\end{array}\right]$, is a quadratic derivation. We note that quadratic derivations and ring derivations are different.

Arriola and Beyer in [16] initiated the stability of functional equations in non-Archimedean spaces. In fact they established the stability of Cauchy functional equations over $p$-adic fields. After their results, some papers (see for instance [17-24]) on the stability of other equations in such spaces have been published. Although different methods are known for establishing the stability of functional equations, almost all proofs depend on the Hyers's method in [3]. In 2003, Radu [25] employed the alternative fixed point theorem, due to Diaz and Margolis [26], to prove the stability of the Cauchy additive functional equation. Subsequently, this method was applied to investigate the Hyers-Ulam stability for the Jensen functional equation [27], as well as for the Cauchy functional equation [28], by considering a general control function $\varphi(x, y)$ with suitable properties. Using such an elegant idea, several authors applied the method to investigate the stability of some functional equations, see [29-33].

Recently, Eshaghi and Khodaei [34] considered the following quadratic functional equation:

$$
f(a x+b y)+f(a x-b y)=2 a^{2} f(x)+2 b^{2} f(y)
$$

and proved the Hyers-Ulam stability of the above functional equation in classical Banach spaces. Recently, Eshaghi [35] proved the Hyers-Ulam stability of homomorphisms and derivations on non-Archimedean Banach algebras.

In the present paper, we adopt the idea of Cădariu and Radu [28] to establish the stability of quadratic homomorphisms and quadratic derivations related to the quadratic functional equation (1.1) over non-Archimedean Banach algebras. Some applications of our results in non-Archimedean Banach algebras over $p$-adic numbers will be exhibited.

Now, we recall some notations and basic definitions used later on in the paper.

In 1897, Hensel [36] discovered the $p$-adic numbers as a number theoretical analogue of power series in complex analysis. During the last three decades $p$-adic numbers have gained the interest of physicists for their research, in particular in problems coming from quantum physics, $p$-adic strings and superstrings $[37,38]$. A key property of $p$-adic numbers is that they do not satisfy the Archimedean axiom: For $x, y>0$, there exists $n \in \mathbb{N}$ such that $x<n y$ (see $[39,40])$.

Example 1.1 Let $p$ be a prime number. For any nonzero rational number $a=p^{r} \frac{m}{n}$ such that $m$ and $n$ are coprime to the prime number $p$, define the $p$-adic absolute value $|a|_{p}=$ 
$p^{-r}$. Then $\|$ is a non-Archimedean norm on $\mathbb{Q}$. The completion of $\mathbb{Q}$ with respect to $\|$ is denoted by $\mathbb{Q}_{p}$ and is called the $p$-adic number field. Note that if $p \geq 3$, then $\left|2^{n}\right|=1$ in for each integer $n$.

Let $\mathbb{K}$ denote a field and function (valuation absolute) $|\cdot|$ from $\mathbb{K}$ into $[0, \infty$ ). A nonArchimedean valuation is a function $|\cdot|$ that satisfies the strong triangle inequality; namely, $|x+y| \leq \max \{|x|,|y|\} \leq|x|+|y|$ for all $x, y \in \mathbb{K}$. The associated field $\mathbb{K}$ is referred to as a non-Archimedean field. Clearly, $|1|=|-1|=1$ and $|n| \leq 1$ for all $n \geq 1$. A trivial example of a non-Archimedean valuation is the function $|\cdot|$ taking everything except 0 into 1 and $|0|=0$. We always assume in addition that $|\cdot|$ is nontrivial, i.e., there is a $z \in \mathbb{K}$ such that $|z| \neq 0,1$.

Let $X$ be a linear space over a field $\mathbb{K}$ with a non-Archimedean nontrivial valuation $|\cdot|$. A function $\|\cdot\|: X \rightarrow[0, \infty)$ is said to be a non-Archimedean norm if it is a norm over $\mathbb{K}$ with the strong triangle inequality (ultrametric); namely, $\|x+y\| \leq \max \{\|x\|,\|y\|\}$ for all $x, y \in X$. Then $(X,\|\cdot\|)$ is called a non-Archimedean space. In any such a space a sequence $\left\{x_{n}\right\}_{n \in \mathbb{N}}$ is Cauchy if and only if $\left\{x_{n+1}-x_{n}\right\}_{n \in \mathbb{N}}$ converges to zero. By a complete non-Archimedean space we mean one in which every Cauchy sequence is convergent. A non-Archimedean Banach algebra is a complete non-Archimedean algebra $\mathcal{A}$ which satisfies $\|x y\| \leq\|x\|\|y\|$ for all $x, y \in \mathcal{A}$. For more details the reader is referred to [41, 42].

Let $X$ be a nonempty set and $d: X \times X \rightarrow[0, \infty]$ satisfying: $d(x, y)=0$ if and only if $x=y, d(x, y)=d(y, x)$ and $d(x, z) \leq \max \{d(x, y), d(y, z)\}$ (strong triangle inequality), for all $x, y, z \in X$. Then $(X, d)$ is called a non-Archimedean generalized metric space. $(X, d)$ is called complete if every $d$-Cauchy sequence in $X$ is $d$-convergent.

Using the strong triangle inequality in the proof of the main result of [26], we get to the following result:

Theorem 1.2 (Non-Archimedean Alternative Contraction Principle) If $(\Omega, d)$ is a nonArchimedean generalized complete metric space and $T: \Omega \rightarrow \Omega$ a strictly contractive mapping (that is $d(T(x), T(y)) \leq L d(x, y)$, for all $x, y \in T$ and a Lipschitz constant $L<1)$. Let $x \in \Omega$, then either

(i) $d\left(T^{n}(x), T^{n+1}(x)\right)=\infty$ for all $n \geq 0$, or

(ii) there exists some $n_{0} \geq 0$ such that $d\left(T^{n}(x), T^{n+1}(x)\right)<\infty$ for all $n \geq n_{0}$; the sequence $\left\{T^{n}(x)\right\}$ is convergent to a fixed point $x^{*}$ of $T ; x^{*}$ is the unique fixed point of $T$ in the set

$$
\Lambda=\left\{y \in \Omega: d\left(T^{n_{0}}(x), y\right)<\infty\right\}
$$

and $d\left(y, x^{*}\right) \leq d(y, T(y))$ for all $y$ in this set.

\section{Non-Archimedean approximately quadratic homomorphisms and quadratic derivations}

Hereafter, unless otherwise stated, we will assume that $\mathcal{A}$ and $\mathcal{B}$ are two non-Archimedean Banach algebras. Also, let $|4|<1$; and we assume that $4 \neq 0$ in $\mathbb{K}$ (i.e., the characteristic of $\mathbb{K}$ is not 4). 
Theorem 2.1 Let $\ell \in\{-1,1\}$ be fixed and let $f: \mathcal{A} \rightarrow \mathcal{B}$ be a mapping with $f(0)=0$ for which there exists a function $\varphi: \mathcal{A}^{4} \rightarrow[0, \infty)$ such that

$$
\left\|f(a x+b y)+f(a x-b y)+f(z w)-2 a^{2} f(x)-2 b^{2} f(y)-f(z) f(w)\right\| \leq \varphi(x, y, z, w)
$$

for all $x, y, z, w \in \mathcal{A}$ and nonzero fixed integers $a, b$. If there exists an $L<1$ such that

$$
\varphi(x, y, z, w) \leq|4|^{\ell(\ell+2)} L \varphi\left(\frac{x}{2^{\ell}}, \frac{y}{2^{\ell}}, \frac{z}{2^{\ell}}, \frac{w}{2^{\ell}}\right)
$$

for all $x, y, z, w \in \mathcal{A}$. Then there exists a unique quadratic homomorphism $\mathcal{H}: \mathcal{A} \rightarrow \mathcal{B}$ such that

$$
\|f(x)-\mathcal{H}(x)\| \leq \frac{L^{\frac{1-\ell}{2}}}{|4|} \psi(x)
$$

for all $x \in \mathcal{A}$, where

$$
\begin{aligned}
\psi(x):= & \max \left\{\varphi\left(\frac{x}{a}, \frac{x}{b}, 0,0\right), \varphi\left(\frac{x}{a}, 0,0,0\right), \frac{1}{\left|2 b^{2}\right|} \varphi(x, x, 0,0),\right. \\
& \left.\frac{1}{\left|2 b^{2}\right|} \varphi(x,-x, 0,0), \varphi\left(0, \frac{x}{b}, 0,0\right)\right\} .
\end{aligned}
$$

Proof Letting $z=w=0$ in (2.1), we get

$$
\left\|f(a x+b y)+f(a x-b y)-2 a^{2} f(x)-2 b^{2} f(y)\right\| \leq \varphi(x, y, 0,0)
$$

for all $x, y \in \mathcal{A}$. Setting $y=-y$ in (2.4), we get

$$
\left\|f(a x-b y)+f(a x+b y)-2 a^{2} f(x)-2 b^{2} f(-y)\right\| \leq \varphi(x,-y, 0,0)
$$

for all $x, y \in \mathcal{A}$. It follows from (2.4) and (2.5) that

$$
\left\|2 b^{2} f(y)-2 b^{2} f(-y)\right\| \leq \max \{\varphi(x, y, 0,0), \varphi(x,-y, 0,0)\}
$$

for all $x, y \in \mathcal{A}$. Putting $y=0$ in (2.4), we get

$$
\left\|2 f(a x)-2 a^{2} f(x)\right\| \leq \varphi(x, 0,0,0)
$$

for all $x \in \mathcal{A}$. Setting $x=0$ in (2.4), we get

$$
\left\|f(b y)+f(-b y)-2 b^{2} f(y)\right\| \leq \varphi(0, y, 0,0)
$$

for all $y \in \mathcal{A}$. Putting $y=b y$ in (2.6), we get

$$
\|f(b y)-f(-b y)\| \leq \max \left\{\frac{1}{\left|2 b^{2}\right|} \varphi(x, b y, 0,0), \frac{1}{\left|2 b^{2}\right|} \varphi(x,-b y, 0,0)\right\}
$$


for all $x, y \in \mathcal{A}$. It follows from (2.8) and (2.9) that

$$
\left\|2 f(b y)-2 b^{2} f(y)\right\| \leq \max \left\{\frac{1}{\left|2 b^{2}\right|} \varphi(x, b y, 0,0), \frac{1}{\left|2 b^{2}\right|} \varphi(x,-b y, 0,0), \varphi(0, y, 0,0)\right\}
$$

for all $x, y \in \mathcal{A}$. Replacing $x$ and $y$ by $\frac{x}{a}$ and $\frac{x}{b}$ in (2.4), respectively, we get

$$
\left\|f(2 x)-2 a^{2} f\left(\frac{x}{a}\right)-2 b^{2} f\left(\frac{x}{b}\right)\right\| \leq \varphi\left(\frac{x}{a}, \frac{x}{b}, 0,0\right)
$$

for all $x \in \mathcal{A}$. Setting $x=\frac{x}{a}$ in (2.7), we get

$$
\left\|2 a^{2} f\left(\frac{x}{a}\right)-2 f(x)\right\| \leq \varphi\left(\frac{x}{a}, 0,0,0\right)
$$

for all $x \in \mathcal{A}$. Putting $y=\frac{x}{b}$ in (2.10), we get

$$
\begin{aligned}
& \left\|2 b^{2} f\left(\frac{x}{b}\right)-2 f(x)\right\| \\
& \leq \max \left\{\frac{1}{\left|2 b^{2}\right|} \varphi(x, x, 0,0), \frac{1}{\left|2 b^{2}\right|} \varphi(x,-x, 0,0), \varphi\left(0, \frac{x}{b}, 0,0\right)\right\}
\end{aligned}
$$

for all $x \in \mathcal{A}$. It follows from (2.11), (2.12) and (2.13) that

$$
\|f(2 x)-4 f(x)\| \leq \psi(x)
$$

for all $x \in \mathcal{A}$. For every $g, h: \mathcal{A} \rightarrow \mathcal{B}$, define

$$
d(g, h):=\inf \{C \in(0, \infty):\|g(x)-h(x)\| \leq C \psi(x), \forall x \in \mathcal{A}\} .
$$

Hence, $d$ defines a complete generalized non-Archimedean metric on $\Omega:=\{g \mid g: \mathcal{A} \rightarrow$ $\mathcal{B}, g(0)=0\}$ (see [27, 28, 33]). Let $T: \Omega \rightarrow \Omega$ be defined by $\operatorname{Tg}(x)=\frac{1}{4^{\ell}} g\left(2^{\ell} x\right)$ for all $x \in \mathcal{A}$ and all $g \in \Omega$. If for some $g, h \in \Omega$ and $C>0$,

$$
\|g(x)-h(x)\| \leq C \psi(x)
$$

for all $x \in \mathcal{A}$, then

$$
\|\operatorname{Tg}(x)-T h(x)\| \leq \frac{1}{|4|^{\ell}}\left\|g\left(2^{\ell} x\right)-h\left(2^{\ell} x\right)\right\| \leq \frac{C}{|4|^{\ell}} \psi\left(2^{\ell} x\right) \leq L C \psi(x)
$$

for all $x \in \mathcal{A}$, so

$$
d(T g, T h) \leq L d(g, h)
$$

Hence $T$ is a strictly contractive mapping on $\Omega$ with the Lipschitz constant $L$. It follows from (2.14) by using (2.2) that

$$
\left\|f(x)-4 f\left(\frac{x}{2}\right)\right\| \leq \psi\left(\frac{x}{2}\right) \leq \frac{L}{|4|} \psi(x)
$$


and

$$
\left\|f(x)-\frac{1}{4} f(2 x)\right\| \leq \frac{1}{|4|} \psi(x)
$$

for all $x \in \mathcal{A}$, that is, $d(f, T f) \leq \frac{\frac{1-\ell}{2}}{|4|}<\infty$.

Now, by the non-Archimedean alternative contraction principle, $T$ has a unique fixed point $\mathcal{H}: \mathcal{A} \rightarrow \mathcal{B}$ in the set $\Lambda=\{g \in \Omega: d(f, g)<\infty\}$, which $\mathcal{H}$ is defined by

$$
\mathcal{H}(x)=\lim _{n \rightarrow \infty} T^{n} f(x)=\lim _{n \rightarrow \infty} \frac{1}{4^{\ell n}} f\left(2^{\ell n} x\right)
$$

for all $x \in \mathcal{A}$. By (2.2),

$$
\lim _{n \rightarrow \infty} \frac{1}{|4|^{\ell(\ell+2) n}} \varphi\left(2^{\ell n} x, 2^{\ell n} y, 2^{\ell n} z, 2^{\ell n} w\right)=0
$$

for all $x, y, z, w \in \mathcal{A}$. It follows from (2.1), (2.15) and (2.16) that

$$
\begin{aligned}
& \left\|\mathcal{H}(a x+b y)+\mathcal{H}(a x-b y)-2 a^{2} \mathcal{H}(x)-2 b^{2} \mathcal{H}(y)\right\| \\
& \quad=\lim _{n \rightarrow \infty} \frac{1}{|4|^{\ell n}}\left\|f\left(2^{\ell n} a x+2^{\ell n} b y\right)+f\left(2^{\ell n} a x-2^{\ell n} b y\right)-2 a^{2} f\left(2^{\ell n} x\right)-2 b^{2} f\left(2^{\ell n} y\right)\right\| \\
& \leq \lim _{n \rightarrow \infty} \frac{1}{|4|^{\ell n}} \varphi\left(2^{\ell n} x, 2^{\ell n} y, 0,0\right) \\
& \leq \lim _{n \rightarrow \infty} \frac{1}{|4|^{\ell(\ell+2) n}} \varphi\left(2^{\ell n} x, 2^{\ell n} y, 0,0\right)=0
\end{aligned}
$$

for all $x, y \in \mathcal{A}$. This shows that $\mathcal{H}$ is quadratic. Also,

$$
\begin{aligned}
\|\mathcal{H}(z w)-\mathcal{H}(z) \mathcal{H}(w)\| & =\lim _{n \rightarrow \infty} \frac{1}{|4|^{2 \ell n}}\left\|f\left(4^{\ell n} z w\right)-f\left(2^{\ell n} z\right) f\left(2^{\ell n} w\right)\right\| \\
& \leq \lim _{n \rightarrow \infty} \frac{1}{|4|^{2 \ell n}} \varphi\left(0,0,2^{\ell n} z, 2^{\ell n} w\right) \\
& \leq \lim _{n \rightarrow \infty} \frac{1}{|4|^{\ell(\ell+2) n}} \varphi\left(0,0,2^{\ell n} z, 2^{\ell n} w\right)=0
\end{aligned}
$$

for all $z, w \in \mathcal{A}$. Therefore, $\mathcal{H}$ is a quadratic homomorphism. Moreover, by the nonArchimedean alternative contraction principle,

$$
d(f, \mathcal{H}) \leq d(f, T f) \leq \frac{L^{\frac{1-\ell}{2}}}{|4|}
$$

This implies the inequality (2.3) holds.

Corollary 2.2 Let $\mathcal{A}, \mathcal{B}$ be non-Archimedean Banach algebras over $\mathbb{Q}_{2}, \ell \in\{-1,1\}$ be fixed and $\theta, r$ be non-negative real numbers such that $\ell r>2 \ell(\ell+2)$. Suppose that a mapping $f: \mathcal{A} \rightarrow \mathcal{B}$ satisfies $f(0)=0$ and

$$
\begin{aligned}
& \left\|f(a x+b y)+f(a x-b y)+f(z w)-2 a^{2} f(x)-2 b^{2} f(y)-f(z) f(w)\right\| \\
& \quad \leq \theta\left(\|x\|^{r}+\|y\|^{r}+\|z\|^{r}+\|w\|^{r}\right)
\end{aligned}
$$


for all $x, y, z, w \in \mathcal{A}$, where $a, b$ are positive fixed integers. Then there exists a unique quadratic homomorphism $H: \mathcal{A} \rightarrow \mathcal{B}$ such that

$$
\|f(x)-\mathcal{H}(x)\| \leq 2^{\frac{\ell(4-r)+r}{2}} \theta\|x\|^{r} \begin{cases}4, & \operatorname{gcd}(a, 2)=\operatorname{gcd}(b, 2)=1 \\ \max \left\{1+2^{i r}, 4\right\}, & a=k 2^{i}, \operatorname{gcd}(b, 2)=1 ; \\ \max \left\{1+2^{j r}, 2^{2 j+2}\right\}, & \operatorname{gcd}(a, 2)=1, b=m 2^{j} \\ \max \left\{2^{i r}+2^{j r}, 2^{2 j+2}\right\}, & a=k 2^{i}, b=m 2^{j}\end{cases}
$$

for all $x \in \mathcal{A}$, where $i, j, k, m \geq 1$ are integers and $\operatorname{gcd}(k, 2)=\operatorname{gcd}(m, 2)=1$.

Proof The proof follows from Theorem 2.1, by taking

$$
\varphi(x, y, z, w)=\theta\left(\|x\|^{r}+\|y\|^{r}+\|z\|^{r}+\|w\|^{r}\right)
$$

for all $x, y, z, w \in \mathcal{A}$. Then we choose $L=2^{\ell(2 \ell+4-r)}$ and we get the desired result.

Corollary 2.3 Let $\mathcal{A}, \mathcal{B}$ be non-Archimedean Banach algebras over $\mathbb{Q}_{2}, \ell \in\{-1,1\}$ be fixed and $\delta$, $s$ be non-negative real numbers such that $\ell s>2 \ell(\ell+2)$. Suppose that a mapping $f: \mathcal{A} \rightarrow \mathcal{B}$ satisfies $f(0)=0$ and

$$
\begin{aligned}
& \left\|f(a x+b y)+f(a x-b y)+f(z w)-2 a^{2} f(x)-2 b^{2} f(y)-f(z) f(w)\right\| \\
& \quad \leq \delta \max \left\{\|x\|^{s},\|y\|^{s},\|z\|^{s},\|w\|^{s}\right\}
\end{aligned}
$$

for all $x, y, z, w \in \mathcal{A}$, where $a, b$ are positive fixed integers. Then there exists a unique quadratic homomorphism $H: \mathcal{A} \rightarrow \mathcal{B}$ such that

$$
\begin{aligned}
& \|f(x)-\mathcal{H}(x)\| \\
& \leq 2^{\frac{\ell(4-s)+s}{2}} \delta\|x\|^{s} \begin{cases}2, & \operatorname{gcd}(a, 2)=\operatorname{gcd}(b, 2)=1 ; \\
\max \left\{2^{i s}, 2\right\}, & a=k 2^{i}, \operatorname{gcd}(b, 2)=1 ; \\
\max \left\{2^{j s}, 2^{2 j+1}\right\}, & \operatorname{gcd}(a, 2)=1, b=m 2^{j} \vee a=k 2^{i}, b=m 2^{j}(j \geq i) ; \\
\max \left\{2^{i s}, 2^{2 j+1}\right\}, & a=k 2^{i}, b=m 2^{j}(i \geq j) ;\end{cases}
\end{aligned}
$$

for all $x \in \mathcal{A}$, where $i, j, k, m \geq 1$ are integers and $\operatorname{gcd}(k, 2)=\operatorname{gcd}(m, 2)=1$.

Theorem 2.4 Let $\ell \in\{-1,1\}$ be fixed and let $f: \mathcal{A} \rightarrow \mathcal{A}$ be a mapping with $f(0)=0$ if $\ell=-1$, for which there exists a function $\varphi: \mathcal{A}^{4} \rightarrow[0, \infty)$ satisfying $(2.2)$ and

$$
\begin{aligned}
& \left\|f(a x+b y)+f(a x-b y)+f(z w)-2 a^{2} f(x)-2 b^{2} f(y)-z^{2} f(w)-f(z) w^{2}\right\| \\
& \quad \leq \varphi(x, y, z, w)
\end{aligned}
$$

for all $x, y, z, w \in \mathcal{A}$ and nonzero fixed integers $a, b$. Then there exists a unique quadratic derivation $\mathcal{D}: \mathcal{A} \rightarrow \mathcal{A}$ such that

$$
\|f(x)-\mathcal{D}(x)\| \leq \frac{L^{\frac{1-\ell}{2}}}{|4|} \psi(x)
$$

for all $x \in \mathcal{A}$, where $\psi(x)$ is defined as in Theorem 2.1. 
Proof By (2.2), if $\ell=1$, we obtain $\varphi(0,0,0,0)=0$. Letting $x=y=z=w=0$ in (2.17), we get $f(0) \leq \varphi(0,0,0,0)$. So $f(0)=0$ for $\ell=1$.

By the same reasoning as that in the proof of Theorem 2.1, there exists a unique quadratic mapping $\mathcal{D}: \mathcal{A} \rightarrow \mathcal{A}$ satisfying (2.18). The mapping $\mathcal{D}: \mathcal{A} \rightarrow \mathcal{A}$ is given by $\mathcal{D}(x)=\lim _{n \rightarrow \infty} T^{n} f(x)=\lim _{n \rightarrow \infty} \frac{1}{4^{\ell n}} f\left(2^{\ell n} x\right)$ for all $x \in \mathcal{A}$. It follows from (2.17) that

$$
\begin{aligned}
& \left\|\mathcal{D}(z w)-z^{2} \mathcal{D}(w)-\mathcal{D}(z) w^{2}\right\| \\
& \quad=\lim _{n \rightarrow \infty} \frac{1}{|4|^{2 \ell n}}\left\|f\left(4^{\ell n} z w\right)-\left(2^{\ell n} z\right)^{2} f\left(2^{\ell n} w\right)-f\left(2^{\ell n} z\right)\left(2^{\ell n} w\right)^{2}\right\| \\
& \quad \leq \lim _{n \rightarrow \infty} \frac{1}{|4|^{2 \ell n}} \varphi\left(0,0,2^{\ell n} z, 2^{\ell n} w\right) \\
& \quad \leq \lim _{n \rightarrow \infty} \frac{1}{|4|^{\ell(\ell+2) n}} \varphi\left(0,0,2^{\ell n} z, 2^{\ell n} w\right)=0
\end{aligned}
$$

for all $z, w \in \mathcal{A}$. Therefore $\mathcal{D}: \mathcal{A} \rightarrow \mathcal{A}$ is a quadratic derivation satisfying (2.18).

Very recently, J. M. Rassias [43] considered the Cauchy difference controlled by the product and sum of powers of norms, that is, $\theta\left\{\|x\|^{p}\|y\|^{p}+\left(\|x\|^{2 p}+\|y\|^{2 p}\right)\right\}$.

Corollary 2.5 Let $\mathcal{A}$ be a non-Archimedean Banach algebra over $\mathbb{Q}_{2}, \ell \in\{-1,1\}$ be fixed and $\varepsilon, p, q$ be non-negative real numbers such that $\ell(p+q)>2 \ell(\ell+2)$. Suppose that a mapping $f: \mathcal{A} \rightarrow \mathcal{A}$ satisfies

$$
\begin{aligned}
& \left\|f(a x+b y)+f(a x-b y)+f(z w)-2 a^{2} f(x)-2 b^{2} f(y)-z^{2} f(w)-f(z) w^{2}\right\| \\
& \quad \leq \varepsilon\left(\|x\|^{p}\|y\|^{q}+\|z\|^{p}\|w\|^{q}\right)
\end{aligned}
$$

for all $x, y, z, w \in \mathcal{A}$, where $a, b$ are positive fixed integers. Then there exists a unique quadratic derivation $\mathcal{D}: \mathcal{A} \rightarrow \mathcal{A}$ such that

$$
\begin{aligned}
& \|f(x)-\mathcal{D}(x)\| \\
& \leq 2^{\frac{\ell(4-p-q)+p+q}{2}} \varepsilon\|x\|^{p+q} \begin{cases}2, & \operatorname{gcd}(a, 2)=\operatorname{gcd}(b, 2)=1 \\
\max \left\{2^{i p}, 2\right\}, & a=k 2^{i}, \operatorname{gcd}(b, 2)=1 ; \\
\max \left\{2^{j q}, 2^{2 j+1}\right\}, & \operatorname{gcd}(a, 2)=1, b=m 2^{j} \\
\max \left\{2^{i p+j q}, 2^{2 j+1}\right\}, & a=k 2^{i}, b=m 2^{j}\end{cases}
\end{aligned}
$$

for all $x \in \mathcal{A}$, where $i, j, k, m \geq 1$ are integers and $\operatorname{gcd}(k, 2)=\operatorname{gcd}(m, 2)=1$.

Competing interests

The authors declare that they have no competing interests.

Authors' contributions

All authors read and approved the final manuscript.

\section{Author details}

${ }^{1}$ Department of Mathematics, Semnan University, P.O. Box 35195-363, Semnan, Iran. ${ }^{2}$ Center of Excellence in Nonlinear Analysis and Applications (CENAA), Semnan University, Semnan, Iran. ${ }^{3}$ Department of Mathematics, Research Institute for Natural Sciences, Hanyang University, Seoul, 133-791, Republic of Korea. 


\section{References}

1. Pólya, G, Szegő, G: Aufgaben und Lehrsätze aus der Analysis, vol. I. Springer, Berlin (1925)

2. Ulam, SM: A Collection of Mathematical Problems. Interscience, New York (1960)

3. Hyers, DH: On the stability of the linear functional equation. Proc. Natl. Acad. Sci. USA 27, $222-224$ (1941)

4. Aoki, T: On the stability of the linear transformation in Banach spaces. J. Math. Soc. Jpn. 2, 64-66 (1950)

5. Rassias, TM: On the stability of the linear mapping in Banach spaces. Proc. Am. Math. Soc. 72, 297-300 (1978)

6. Găvruta, P: A generalization of the Hyers-Ulam-Rassias stability of approximately additive mappings. J. Math. Anal. Appl. 184, 431-436 (1994)

7. Rassias, JM: On approximation of approximately linear mappings by linear mappings. J. Funct. Anal. 46, 126-130 (1982)

8. Rassias, JM: Solution of a problem of Ulam. J. Approx. Theory 57(3), 268-273 (1989)

9. Rassias, JM: On the stability of the Euler-Lagrange functional equation. Chin. J. Math. 20(2), 185-190 (1992)

10. Rassias, JM: Complete solution of the multi-dimensional problem of Ulam. Discuss. Math. 14, 101-107 (1994)

11. Găvruta, P: An answer to a question of John M. Rassias concerning the stability of Cauchy equation. In: Advances in Equations and Inequalities. Hardronic Math. Ser., pp. 67-71 (1999)

12. Bourgin, DG: Approximately isometric and multiplicative transformations on continuous function rings. Duke Math. J. $16,385-397$ (1949)

13. Badora, R: On approximate ring homomorphisms. J. Math. Anal. Appl. 276, 589-597 (2002)

14. Šemrl, P: The functional equation of multiplicative derivation is superstable on standard operator algebras. Integral Equ. Oper. Theory 18, 118-122 (1994)

15. Badora, R: On approximate derivations. Math. Inequal. Appl. 9, 167-173 (2006)

16. Arriola, LM, Beyer, WA: Stability of the Cauchy functional equation over $p$-adic fields. Real Anal. Exch. 31, 125-132 $(2005 / 2006)$

17. Cho, YJ, Park, C, Saadati, R: Functional inequalities in non-Archimedean Banach spaces. Appl. Math. Lett. 23, 1238-1242 (2010)

18. Cho, YJ, Saadati, R: Lattice non-Archimedean random stability of ACQ functional equation. Adv. Differ. Equ. 2011, 31 (2011)

19. Cho, YJ, Saadati, R, Vahidi, J: Approximation of homomorphisms and derivations on non-Archimedean Lie $C^{*}$-algebras via fixed point method. Discrete Dyn. Nat. Soc. 2012, Article ID 373904 (2012)

20. Eshaghi Gordji, M, Khodabakhsh, R, Khodaei, H, Park, C, Shin, DY: A functional equation related to inner product spaces in non-Archimedean normed spaces. Adv. Differ. Equ. 2011, 37 (2011)

21. Eshaghi Gordji, M, Khodaei, H, Khodabakhsh, R: General quartic-cubic-quadratic functional equation in non-Archimedean normed spaces. U. P. B. Sci. Bull., Ser. A 72, 69-84 (2010)

22. Eshaghi Gordji, M, Savadkouhi, MB: Stability of cubic and quartic functional equations in non-Archimedean spaces. Acta Appl. Math. 110, 1321-1329 (2010)

23. Eshaghi Gordji, M, Savadkouhi, MB: Stability of a mixed type cubic-quartic functional equation in non-Archimedean spaces. Appl. Math. Lett. 23, 1198-1202 (2010)

24. Najati, A, Cho, YJ: Generalized Hyers-Ulam stability of the pexiderized Cauchy functional equation in non-Archimedean spaces. Fixed Point Theory Appl. 2011, Article ID 309026 (2011)

25. Radu, $\mathrm{V}$ : The fixed point alternative and the stability of functional equations. Fixed Point Theory 4, 91-96 (2003)

26. Diaz, JB, Margolis, B: A fixed point theorem of the alternative for contractions on the generalized complete metric space. Bull. Am. Math. Soc. 126, 305-309 (1968)

27. Cădariu, L, Radu, V: Fixed points and the stability of Jensen's functional equation. J. Inequal. Pure Appl. Math. 4, Article ID 4 (2003)

28. Cădariu, L, Radu, V: On the stability of the Cauchy functional equation: A fixed point approach. Grazer Math. Ber. 346, 43-52 (2004)

29. Cădariu, L, Radu, V: Fixed point methods for the generalized stability of functional equations in a single variable. Fixed Point Theory Appl. 2008, Article ID 749392 (2008)

30. Cho, YJ, Kang, Jl, Saadati, R: Fixed points and stability of additive functional equations on the Banach algebras. J. Comput. Anal. Appl. 14, 1103-1111 (2012)

31. Găvruta, P, Găvruta, L: A new method for the generalized Hyers-Ulam-Rassias stability. Int. J. Nonlinear Anal. Appl. 1(2), 11-18 (2010)

32. Jung, S, Kim, T: A fixed point approach to the stability of the cubic functional equation. Bol. Soc. Mat. Mexicana 12 , 51-57 (2006)

33. Mirmostafaee, AK: Hyers-Ulam stability of cubic mappings in non-Archimedean normed spaces. Kyungpook Math. J. 50, 315-327 (2010)

34. Eshaghi Gordji, M, Khodaei, $\mathrm{H}$ : On the generalized Hyers-Ulam-Rassias stability of quadratic functional equations. Abstr. Appl. Anal. 2009, Article ID 923476 (2009)

35. Eshaghi Gordji, M: Nearly ring homomorphisms and nearly ring derivations on non-Archimedean Banach algebras. Abstr. Appl. Anal. 2010, Article ID 393247 (2010)

36. Hensel, K: Uber eine neue Begrundung der Theorie der algebraischen Zahlen. Jahresber. Dtsch. Math.-Ver. 6, 83-88 (1897)

37. Khrennikov, A: $p$-adic Valued Distributions in Mathematical Physics. Kluwer Academic, Dordrecht (1994)

38. Khrennikov, A: Non-Archimedean Analysis: Quantum Paradoxes, Dynamical Systems and Biological Models. Kluwer Academic, Dordrecht (1997)

39. Gouvêa, FQ: p-adic Numbers. Springer, Berlin (1997)

40. Vladimirov, VS, Volovich, IV, Zelenov, El: $p$-adic Analysis and Mathematical Physics. World Scientific, Singapore (1994)

41. Murphy, GJ: Non-Archimedean Banach algebras. PhD thesis, University of Cambridge (1977)

42. Narici, L: Non-Archimedian Banach spaces and algebras. Arch. Math. 19, 428-435 (1968)

43. Ravi, K, Arunkumar, M, Rassias, JM: Ulam stability for the orthogonally general Euler-Lagrange type functional equation. Int. J. Math. Stat. 3, 36-46 (2008)

44. Bouikhalene, B, Elqorachi, E, Rassias, JM: The superstability of d'Alembert's functional equation on the Heisenberg group. Appl. Math. Lett. 23, 105-109 (2010) 
45. Cao, H-X, Lv, J-R, Rassias, JM: Superstability for generalized module left derivations and generalized module derivations on a Banach module. I. J. Inequal. Appl. 2009, Article ID 718020 (2009)

46. Cao, H-X, LV, J-R, Rassias, JM: Superstability for generalized module left derivations and generalized module derivations on a Banach module. II. J. Inequal. Pure Appl. Math. 10(3), 85 (2009)

doi:10.1186/1687-1847-2012-128

Cite this article as: Eshaghi Gordji et al.: Fixed points and quadratic equations connected with homomorphisms and derivations on non-Archimedean algebras. Advances in Difference Equations 2012 2012:128.

Submit your manuscript to a SpringerOpen ${ }^{\circ}$ journal and benefit from:

- Convenient online submission

- Rigorous peer review

- Immediate publication on acceptance

Open access: articles freely available online

- High visibility within the field

- Retaining the copyright to your article 